Volume 23 (2018) 153-156

DOI: $10.24330 /$ ieja.373657

\title{
PERINORMAL POLYNOMIAL DOMAINS
}

\author{
Tiberiu Dumitrescu and Anam Rani \\ Received: 05 April 2017; Revised: 24 July 2017; Accepted: 01 August 2017 \\ Communicated by Abdullah Harmanc1 \\ Abstract. Let $A$ be a domain. We relate the perinormality (as defined by \\ Epstein and Shapiro) of $A$ and $A[X]$ for a narrow class of Noetherian domains. \\ Mathematics Subject Classification (2010): 13A15, 13F05 \\ Keywords: Perinormal domain, Hilbertian field, pullback
}

\section{Introduction}

In [1] and [2], Epstein and Shapiro studied the integral domains $A$ with the property that every overring $B$ of $A$ which satisfies going down over $A$ is $A$-flat (called by them perinormal domains). Krull domains are typical examples of perinormal domains. [1, Question 3] asks to relate the perinormality of $A$ and $A[X]$. Using the pullback approach from [2], we provide an answer for a narrow class of Noetherian domains. Recall that a field $K$ is Hilbertian if given $f_{i}\left(T_{1}, \ldots, T_{n}, X\right)$ irreducible polynomials in $K\left(T_{1}, \ldots, T_{n}\right)[X], 1 \leq i \leq k$, and $g \in K\left[T_{1}, \ldots, T_{n}\right]-\{0\}$, there exist $a_{1}, \ldots, a_{n} \in K$ such that each $f_{i}\left(a_{1}, \ldots, a_{n}, X\right)$ is defined and irreducible in $K[X]$ and $g\left(a_{1}, \ldots, a_{n}\right) \neq 0$, cf. [3, Chapter 11]. For an ideal $I$ of a ring $A$, denote by $V_{A}(I)$ the Zariski closed set defined by $I$. We use standard terminology like in [4]. Our result is:

Theorem 1.1. Let $A$ be a Noetherian domain with the integral closure $A^{\prime}$. Assume that the conductor $\left(A: A^{\prime}\right)$ has height at least two as an ideal of $A^{\prime}$ and $A /\left(A: A^{\prime}\right)$ is zero-dimensional and not local. Then the first three assertions below are equivalent and imply the fourth one.

(a) $A\left[X_{1}, \ldots, X_{n}\right]$ is perinormal for every $n \geq 0$.

(b) $A\left[X_{1}, \ldots, X_{n}\right]$ is perinormal for some $n \geq 1$.

(c) $A\left(X_{1}, \ldots, X_{n}\right)$ is perinormal for some $n \geq 1$.

(d) A is perinormal.

Moreover, if $A / M$ is a Hilbertian field for every $M \in V_{A}\left(A: A^{\prime}\right)$, then all four assertions are equivalent. 


\section{Lemmata and proof of Theorem 1.1}

The proof is based on two lemmas. In [2, Definition 3.2], an integral extension of rings $A \subseteq B$ is called apparently fragile if for every ring $C$ with $A \subset C \subseteq B$, there exists a minimal prime $P$ of $A$ which is not unibranched in $B$. The extension is called fragile if $A_{P} \subseteq B_{A-P}$ is apparently fragile for every prime ideal $P$ of $A$. Due to Cohen-Seidenberg theorems, it can be seen that, when $A$ is an integrally closed Noetherian domain and $B$ is a finite reduced ring extension of $A$, then $A \subseteq B$ is apparently fragile if and only if there is no domain $C, A \subset C \subseteq B$.

Lemma 2.1. Let $A \subseteq C$ and $B \subseteq D$ be integral ring extensions. Then $A \times B \subseteq$ $C \times D$ is fragile if and only if $A \subseteq C$ and $B \subseteq D$ are fragile.

Proof. The assertion follows combining the following simple facts. The extension $A \times B \subseteq C \times D$ is integral, $\operatorname{Spec}(A \times B)$ is the disjoint union of $\operatorname{Spec}(A)$ and $\operatorname{Spec}(B)$, and, if $P \in \operatorname{Spec}(A)$, then the extension $(A \times B)_{P \times B} \subseteq(C \times D)_{(A-P) \times B}$ is isomorphic to $A_{P} \subseteq C_{A-P}$.

In the sequel, if $A$ is a ring and $B_{1}, \ldots, B_{k}$ are ring extensions of $A$, we embed diagonally $A$ in $\prod_{i=1}^{k} B_{i}$ and simply write $A \subseteq \prod_{i=1}^{k} B_{i}$.

Lemma 2.2. Let $K$ be a field and $L_{1}, \ldots, L_{k}$ finite field extensions of $K$. Then the first three assertions below are equivalent and imply the fourth one.

(a) $K\left[X_{1}, \ldots, X_{n}\right] \subseteq \prod_{i=1}^{k} L_{i}\left[X_{1}, \ldots, X_{n}\right]$ is fragile for every $n \geq 1$.

(b) $K\left(X_{1}, \ldots, X_{n}\right) \subseteq \prod_{i=1}^{k} L_{i}\left(X_{1}, \ldots, X_{n}\right)$ is fragile for every $n \geq 1$.

(c) $K\left(X_{1}, \ldots, X_{n}\right) \subseteq \prod_{i=1}^{k} L_{i}\left(X_{1}, \ldots, X_{n}\right)$ is fragile for some $n \geq 1$.

(d) $K \subseteq \prod_{i=1}^{k} L_{i}$ is fragile.

Moreover, if $K$ is a Hilbertian field, then all four assertions are equivalent.

Proof. Note that due to [2, Proposition 3.8], we may change everywhere fragile by apparently fragile. The case $k=1$ is obvious, so we may suppose that $k \geq 2$. Set $A=K\left[X_{1}, \ldots, X_{n}\right], S=A-\{0\}, B=\prod_{i=1}^{k} L_{i}\left[X_{1}, \ldots, X_{n}\right], C=\prod_{i=1}^{k} L_{i}$ and observe that we have $B=C\left[X_{1}, \ldots, X_{n}\right], A_{S}=K\left(X_{1}, \ldots, X_{n}\right)$ and $B_{S}=$ $\prod_{i=1}^{k} L_{i}\left(X_{1}, \ldots, X_{n}\right)$, because $A \subseteq B$ is finite. $(a) \Rightarrow(b)$ Deny; so there exists a field $E$ situated strictly between $A_{S}$ and $B_{S}$. By $(a)$, we get $E \cap B=A$. We obtain $E=(E \cap B)_{S}=A_{S}$, a contradiction. $(b) \Rightarrow(c)$ is trivial. $(c) \Rightarrow(d)$ Deny; hence there exists a field $M$ situated strictly between $K$ and $C$, so $M\left(X_{1}, \ldots, X_{n}\right)$ is situated strictly between $A_{S}$ and $B_{S}$, a contradiction.

Now we prove $(d) \Rightarrow(b)$ for $K$ being a Hilbertian field. Suppose that $(b)$ fails. Then there exists a field $E$ situated strictly between $A_{S}$ and $B_{S}$. We may assume 
$E=A_{S}(\alpha)$ for some $\alpha=\left(\alpha_{1}, \ldots, \alpha_{n}\right) \in B_{S}-A_{S}$. Here $\alpha_{i}=\alpha_{i}\left(X_{1}, \ldots, X_{n}\right) \in$ $L_{i}\left(X_{1}, \ldots, X_{n}\right)$ and let $p \in S$ such that $p \alpha_{i} \in B$ for each $i$. It follows that the minimal polynomial $f\left(X_{1}, \ldots, X_{n}, Y\right) \in A_{S}[Y]$ of $\alpha$ over $A_{S}$ equals the minimal polynomial of $\alpha_{i}$ over $A_{S}$ for each $i$ between 1 and $k$. As $K$ is Hilbertian, there exist $a_{1}, \ldots, a_{n} \in K$ such that $p\left(a_{1}, \ldots, a_{n}\right) \neq 0$ and $g=f\left(a_{1}, \ldots, a_{n}, Y\right)$ is defined and irreducible in $K[Y]$. Let $\beta_{i}=\alpha_{i}\left(a_{1}, \ldots, a_{n}\right)$ and $\beta=\left(\beta_{1}, \ldots, \beta_{n}\right) \in C$. As $g$ is irreducible and $g(\beta)=0$, it follows that $g$ is the minimal polynomial of $\beta$ over $K$, hence $K(\beta)$ is a field situated strictly between $K$ and $C$. The implication $(c) \Rightarrow(b)$ follows from the fact that, for $n \geq 1, K\left(X_{1}, \ldots, X_{n}\right)$ is Hilbertian [3, Theorem 12.10] and from implications $(c) \Rightarrow(d)$ and $(d) \Rightarrow(b)$ (for $K$ Hilbertian) proved above. $(b) \Rightarrow(a)$ Let $D$ be a domain situated between $A$ and $B$. By $(b)$, we get $A_{S}=D_{S}$, hence $D \subseteq A_{S} \cap B=A$, thus $D=A$.

Proof. Proof of Theorem 1.1. $(a) \Rightarrow(b)$ and $(a) \Rightarrow(d)$ are trivial and $(b) \Rightarrow$ (c) follows from the fact that perinormality is a local property [1, Proposition 2.5]. $(c) \Rightarrow(a)$ Set $B=A\left(X_{1}, \ldots, X_{n}\right), B^{\prime}=A^{\prime}\left(X_{1}, \ldots, X_{n}\right)$ and $I=(A$ : $\left.A^{\prime}\right)$. As $A \subseteq A^{\prime}$ is finite, we have that $B^{\prime}=B \otimes_{A} A^{\prime}$ is the integral closure of $B$. Note that $I B$ is also an ideal of $B^{\prime}$. Since $A / I$ is zero-dimensional, it follows that $B / I B=(A / I)\left(X_{1}, \ldots, X_{n}\right) \subseteq B^{\prime} / I B=\left(A^{\prime} / I\right)\left(X_{1}, \ldots, X_{n}\right)$ are zero-dimensional rings. Since $B$ is perinormal, it follows that $B / I B \subseteq B^{\prime} / I B$ is fragile [2, Theorem 3.13] and $I$ is a radical ideal of $A$, cf. [2, Lemma 3.6]. Thus $A / I \subseteq A^{\prime} / I$ is isomorphic to a direct product of finite extensions $K_{i} \subseteq$ $\prod_{j=1}^{k_{i}} L_{i j}, 1 \leq i \leq l$, where $V_{A}(I)=\left\{M_{1}, \ldots, M_{l}\right\}, K_{i}=A / M_{i}, i=1, \ldots, l$ and $\left\{L_{i 1}, \ldots, L_{i k_{i}}\right\}=\left\{A^{\prime} / N \mid N \in V_{A^{\prime}}(I), N \cap A=M_{i}\right\}$. Then the fragile extension $B / I B \subseteq B^{\prime} / I B$ is isomorphic to the direct product of extensions $K_{i}\left(X_{1}, \ldots, X_{n}\right) \subseteq$ $\prod_{j=1}^{k_{i}} L_{i j}\left(X_{1}, \ldots, X_{n}\right)$, so all these extensions are fragile, cf. Lemma 2.1. Let $m \geq 0$ and set $C=A\left[X_{1}, \ldots, X_{m}\right], C^{\prime}=A^{\prime}\left[X_{1}, \ldots, X_{m}\right]$. By Lemma 2.2, all extensions $K_{i}\left[X_{1}, \ldots, X_{m}\right] \subseteq \prod_{j=1}^{k_{i}} L_{i j}\left[X_{1}, \ldots, X_{m}\right]$ are fragile, hence so is their product which is isomorphic to $C / I C \subseteq C^{\prime} / I C$. By [2, Theorem 3.5], $C$ is perinormal. For the "moreover" part, if $(d)$ holds and $A / M$ is Hilbertian for all $M \in V_{A}\left(A: A^{\prime}\right)$, we can repeat the preceding proof to get that $(a)$ holds.

Acknowledgment. The first author gratefully acknowledges the warm hospitality of ASSMS Govt. Coll. University Lahore during his visits between 2006 and 2017. The second author is highly grateful to ASSMS Govt. Coll. University Lahore, Pakistan in supporting and facilitating this research. 


\section{References}

[1] N. Epstein and J. Shapiro, Perinormality-a generalization of Krull domains, J. Algebra, 451 (2016), 65-84.

[2] N. Epstein and J. Shapiro, Perinormality in pullbacks, to appear in J. Commut. Algebra, https://projecteuclid.org/euclid.jca/1491379230, arXiv:1511.06473v2 [math.AC].

[3] M. D. Fried and M. Jarden, Field Arithmetic, Results in Mathematics and Related Areas (3), 11, Springer-Verlag, Berlin, 1986.

[4] R. Gilmer, Multiplicative Ideal Theory, Pure and Applied Mathematics, 12, Marcel Dekker, Inc., New York, 1972.

\section{Tiberiu Dumitrescu}

Facultatea de Matematica si Informatica

University of Bucharest

14 Academiei Str., Bucharest

RO 010014, Romania

e-mail: tiberiu@fmi.unibuc.ro, tiberiu_dumitrescu2003@yahoo.com

Anam Rani (Corresponding Author)

Abdus Salam School of Mathematical Sciences

GC University, Lahore

68-B, New Muslim Town

Lahore 54600, Pakistan

e-mail: anamrane@gmail.com 